\title{
Image-Forming System Design of Dynamic Targets Based on Reflecting Mirror Splicing
}

\author{
Hong Mei Zhu ${ }^{1, a}$, Yan Piao ${ }^{1}$

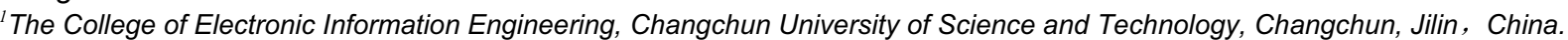

\begin{abstract}
In this paper, the author designed an area-scan CCD reflecting mirror splicing image-forming system with area-scan CCD ICX415AL as its transducer module and this system can be used for tracking dynamic targets. By analyzing the theory of vignetting generating, the author made mathematic model of vignetting and confirmed the splicing and overlapping pixel number of the optical system. What's more, the sequential circuit and driving power circuit of ICX415ALwas designed and the correlated noise in video signals was strained with CDS technology. Therefore, the signal-to-noise ratio (SNR) of the system was elevated. With FPGA as its core controlling module, this system postponed the splicing image-forming system to a period during which a line of CCD data are read, thus the need of real-time tracking was completely met.
\end{abstract}

Keywords: reflecting mirror, area-scan CCD, dynamic targets, FPGA. .

\section{Introduction}

As for the tracking of dynamic targets, the image-forming system with big viewing field and high resolution can greatly increase the probability of target acquisition and recognition[1]. Therefore, most dynamic target tracking systems adopt area-scan CCD as their image-forming module. However, with the rising number of $\mathrm{CCD}$ pixels, frame frequency decreases inevitably, and the accurate tracking of dynamic targets fails. Thus, the method of splicing many minor area-scan CCDs can guarantee the high frame frequency in accurate tracking and at the same time meet the need of big viewing field and high resolution. At present, there are a lot of area-scan CCD splicing methods, such as mechanical splicing, multiple-lens splicing, prism splicing, reflecting mirror splicing and so on. Among them, CCD reflecting mirror splicing uses reflecting spectroscope to divide one image plane into two or more spaced planes. Then, many CCDs can be interlacedly placed on every plane and the pixels of the two interlaced neighboring CCD border are made to meet overlapping requirement. Thus, an equivalent big viewing-field detector is formed.

\section{Formation of the mathematic model of vignetting}

For Point A and Point B which form images in the infinite distance on reflecting surface $\mathrm{CCD}$, when they pass through the optical system, they are supposed to converge at image points $A^{\prime}$ and $B^{\prime}$ on the reflecting surface. But, for Point A and Point B, with the existence of reflecting mirror on the optical path, part of their rays haven't been reflected by the reflecting mirror and instead they are projected directly behind the reflecting mirror. For Point A, this part of optical energy is lost and this can be reflected on the image by the fact that the digital value of this point is darker. When the position of the reflecting mirror and that of the plane on which CCD image plane is located are fixed, it can be known through geometrical relationship that for any point whose viewing angle is larger than B's angle $\omega$, all their rays can be reflected on the above reflecting surface CCD through the reflecting mirror. Therefore, no energy is lost and all the points whose viewing angles are larger than Angle $\omega$ can be considered to have created no vignetting.

According to cosine theorem and the known conditions, we can get the values of Angles $\alpha$ and $\beta$ as is shown in the following formula:

$\cos \alpha=\sqrt{\left(2 f d+\Delta a D+2 \Delta a^{2}\right) /\left(4 f^{2}+4 \Delta a^{2}+4 \Delta a D+D^{2}\right)}$

$\cos \beta=\frac{\sqrt{\left(4 f^{2}-D^{2}+4 \Delta a^{2}\right) /\left(4 f^{2}+D^{2}-4 \Delta a^{2}\right)}}{\sqrt{4 f^{2}+4 \Delta a^{2}+4 a D+D^{2}}}$

Here $\mathrm{D}$ is the caliber of the optical system, $f$ is the focal length of the optical system, ${ }^{d_{0}}$ is the distance from reflecting mirror to image plane, $\Delta a$ is the distance from vignetting point on the image plane to the centre of viewing field.

The energy of a point light source distributes on an image plane as a round spot. For image-forming Point $A^{\prime}$ on the reflecting surface, the energy that a reflecting surface $\mathrm{CCD}$ receives should include all the energy of the entire light spot. However, the energy within Angle $\alpha$ has not been reflected. Therefore, the energy of Point $A^{\prime}$ can only be the difference between the energy of the light spot and that of the area within

${ }^{\mathrm{a} C}$ Corresponding author: custzhm@126.com 
Angle $\alpha$. As can be shown in Figure2, when the position of the optical system and that of the CCD image plane $l$ are fixed, the size of the light spot created by the point light source is fixed. Suppose that the diameter of the round light spot is $\mathrm{d}$, then the ratio of the light spot area of Angel $\alpha$ to that of Angle $\beta$ is

$$
\left\{\frac{d}{4} \arcsin \left[\frac{2 \Delta a\left(l-d_{0}\right)}{d_{0} d}\right]-\sqrt{\left(\frac{d}{4}\right)^{2}-\left(\frac{\Delta a\left(l-d_{0}\right)}{d_{0}}\right)^{2}} \mathrm{~g} \frac{\Delta a\left(l-d_{0}\right)}{d_{0}}\right\}
$$

Suppose $\mathrm{E}$ is the energy of a point in infinite distance, and $E^{\prime}$ is the energy of image-forming Point $A^{\prime}$, then

(4)

$$
E^{\prime}: E=S_{\beta}: S_{W}
$$

$E^{\prime}=E\left\{\frac{d}{4} \arcsin \left[\frac{2 \Delta a\left(l-d_{0}\right)}{d_{0} d}\right]-\sqrt{\left(\frac{d}{4}\right)^{2}-\left(\frac{\Delta a\left(l-d_{0}\right)}{d_{0}}\right)^{2}} \mathrm{~g} \frac{\Delta a\left(l-d_{0}\right)}{d_{0}}\right\}$ (5)

Formula ( 5 ) is the energy value that Point $A^{\prime}$ receives and Point $A^{\prime}$ is a random point within the vignetting area of reflecting surface $C C D$.

The establishment of mathematic model plays a guiding role in splicing optical system. The critical points $\mathrm{B} 1$ and $\mathrm{B} 2$ of the vignetting on the two $\mathrm{CCD}$ image planes can be calculated through the mathematic model, as is shown in Figure 1.

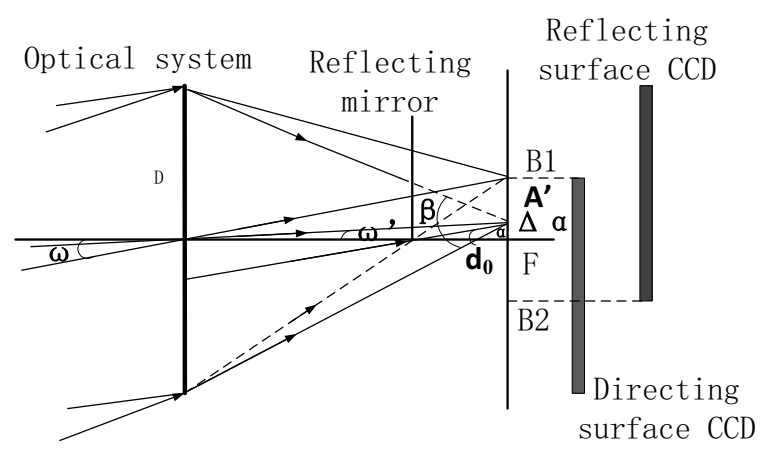

Figure 1: diagram of the production of vignetting of reflecting surface CCD

The reflecting surface CCD is placed on Point B2--the maximal critical point of the direct shooting surface vignetting, while the direct shooting surface CCD is located on Point B1-- the maximal critical point of the reflecting surface vignetting. When the optical energy of the shot target radiates respectively on the direct shooting and reflecting CCDs, a compensation can be made via adding the energy of corresponding pixels. According to calculation, if the optical system of reflecting mirrors is spliced in accordance with overlapping area, 324 pixels will be overlapped and these overlapped pixels will account for $1 / 5$ of the total horizontal pixels. After compensation, an integral radiometric calibration experiment of the system has to be carried out to eliminate the impact of overlapped noise resulted by adding on the image. Through radiometric calibration, not only compensation factor can be confirmed but also the impact of nonuniformity of pixels can be eliminated.

\section{The composition of area-scan CCD image-forming system}

The driving pulses for ICX415AL to produce, transfer and sense charges include vertical transfer clocks $(\Phi \mathrm{V} 1 、 \Phi \mathrm{V} 2 、 \Phi \mathrm{V} 3)$, horizontal transfer clocks $(\Phi H 1 、 \Phi H 2)$, reset clock $(\Phi R G)$ and substrate clock ( (SUB ). Every driving pulse should meet exactly the requirement of phase time sequences in order to guarantee normal operation of CCD. Whenever a set of vertical transfer $\operatorname{clock} \Phi \mathrm{V} 1 、 \Phi \mathrm{V} 2 、 \Phi \mathrm{V} 3$ time sequences are transmitted, the collected charges of every line of pixels of area-scan CCD will move a line to the horizontal shift register; Whenever a set of horizontal transfer clock $\Phi H 1 、 \Phi H 2$ time sequences are transmitted, the charges in the horizontal shift register will transfer to the output terminal once. The transferred charges will be output to the output amplifier where these charges will be converted to the form of voltage and output from OS pins. The reset clock $\Phi R G$ clears away the remaining charges in the horizontal shift register while the substrate clock ( $\Phi$ SUB) determines the adjusting time of CCD shutter. The more substrate clocks ( $\Phi S U B$ ) in a frame, the shorter the exposure time of CCD photosensitive areas.

According to the accuracy detecting result of optical system splicing, the error of straightness and coplanarity of the same horizontal lines is smaller than $1 / 3$ of a pixel. So, every line of data are used as a caching unit. This method doesn't need external RAM chips and it takes up small storage space to use a line as a caching unit. ICX415AL only needs $782 * 10=7820$ bit storage space and FPGA on-chip RAM can meet this need. Meanwhile the caching time is very short. With ping-pong structure reading mode, the caching time is only the period during which a line of data are read by CCD. The advantages of using FPGA on-chip RAM as its caching memory lie in the facts that signal crosstalk caused by the usage of an external memory can be avoided and that the designing requirement for the circuit board is not very high. What's more, the real-time performance of image output is good and it only takes the time for reading a line.

The procedure of ping-pong caching structure is as follows: in the first buffering cycle, the first line of data are written respectively into RAM1 and RAM3; in the second buffering cycle, the data are stored into RAM2 and RAM4 through the switch of writing enabling signals and at the same time, the first line of data are read from RAM1 and RAM3; in the third buffering cycle, the third line of data are written into RAM1 and RAM3 and meanwhile, the second line of data are read from RAM2 and RAM4. In this cycling way, the 
seamless cache and processing of digital streams is fulfilled.

After multiplying the synthesized image data with corresponding adjusting factor of radiometric calibration, the adjusted final output image is obtained as is shown in Figure2 (c) and (d). The original image is

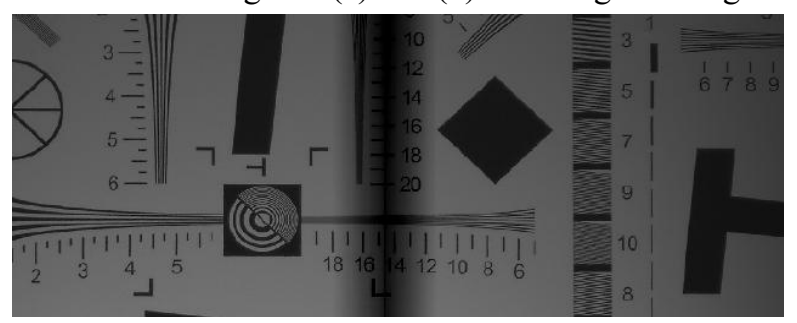

Figure 2-(a): the image of inner calibration

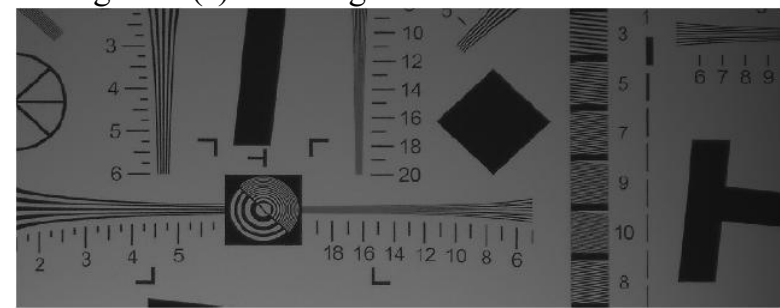

Figure 2 -(c): the image of inner calibration after vignetting is eliminated

As for the effect of image restoration, apart from subjunctive evaluation on resolution with human-being's eyes, objective comparison with image quality evaluating factors can also be made. In this paper, the compensated image is evaluated with signal-to-noise ratio (SNR) of the image ${ }^{[6]}$ and gray mean grads(GMG) with no reference evaluating index. The higher the values of SNR and GMG, the clearer the image and the better the image quality. After selecting the central region of the spliced image and making calculation, we can get the following results as are shown in Chart 1.

Chart 1: comparison of SNR

\begin{tabular}{lllll}
\hline & Fig.1(a) & Fig.1(c) & Fig.1(b) & Fig.1(d) \\
\hline SNR & $12.79 \mathrm{~dB}$ & $27.34 \mathrm{~dB}$ & $13.23 \mathrm{~dB}$ & $28.67 \mathrm{~dB}$ \\
\hline GMG & 4.542 & 7.462 & 4.911 & 7.636 \\
\hline
\end{tabular}

It is obvious that when the image is processed with the above-mentioned vignetting processing method which is based on degradation model, its GMG is greatly elevated. The SNR of the image is elevated at least by $15 \mathrm{~dB}$. The grayscale of the processed image is in uniform distribution and this can be considered as ideal effect.

\section{Summary}

In this paper, the mathematic model of vignetting generating in reflecting mirror splicing optical system is established. With this model, the number of overlapping pixels of the viewing field centre in reflecting mirror splicing system is confirmed and the area-scan CCD image-forming system which can satisfy dynamic target tracking is designed. The compensation for two area-scan CCD data is made in accordance with the number of overlapping pixels. The vignetting in central shown in Figure2（a）and（b). Through comparison, we can conclude that this imaging system can acquire clear images in both indoor roller imaging experiment and outfield imaging experiment and what's more, it can eliminate image degradation caused by vignetting on the premise of satisfying dynamic target tracking.

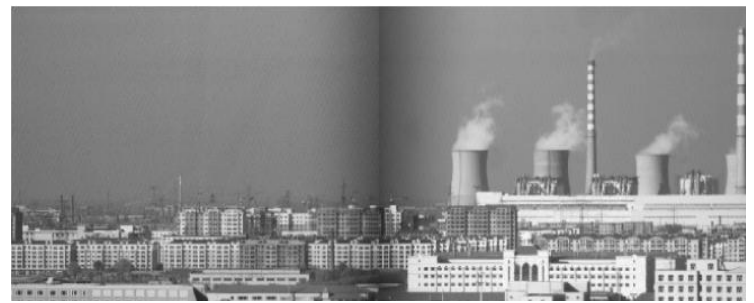

Figure 2-(b): the image of the outfield experiment

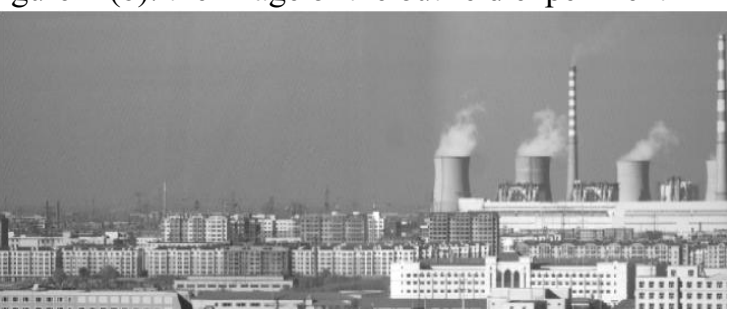

Figure 2-(d): the image of the outfield experiment after vignetting is eliminated

viewing field is eliminated on the premise of meeting real-time tracking need and the SNR of the image is increased at least by $15 \mathrm{~dB}$.

\section{References}

[1] Wang Qingyou. Application Technology of Image Transducer. [M], Beijing: the Electronic Industry Press, 2006: 343-414.

[2] Thomas W.Mccurnin. Signal Processing for Low Level、High Precision CCD Imaging[C]. Proc SPIE, 1991, 1448: 225-235.

[3] Li Guoning, Liu Yanyan, Jin Longxu. The Study of Area-Scan CCD Imaging System for Dynamic Target Tracking. Optical Precision Engineering [J], 2008, 3 . 558-564.

[4] Tong Shoufeng, Ran Jin, Hao Zhihang. The Study of the Technology for Reducing CCD Image Transducer Noise. Optical Precision Engineering [J], 2000,8(2): 140-146.

[5] Xu Xiuzhen, Li Zitian, Cao Lijun.CCD Noise Analysis and Disposing Technology. Infrared and Laser Engineering, 2004,33(4): 343-347.

[6] Tong Shoufeng, Ran Jin, Hao Zhihang. The Analysis of Several CDS Topology Circuits [J], Semiconductor Photoelectronics, 2000, 21(5) : 358-362. 